\title{
報文
}

REGULAR PAPERS

\section{Computer Program TRIO-FIT for Third Order Calculation of Ion Trajectory with Help of Automatic Fitting Function}

\author{
Takekiyo Matsuo*
}

(Received July 5, 198:)

\begin{abstract}
Computer program TRIO FIT was constructed in order to calculate ion trajectory up to third order approximation with the help of automatic fitting function (simplex method). The way how to use TRIO-FIT is explained by using a model mass spectrometer system.
\end{abstract}

\section{Introduction}

The principle of ion optics for a mass spectrometer had been already developed since the Aston's mass spectrograph was constructed. The first order ion optics was completed by the paper of Mattauch and Herzog in 1934. In 1950, the second order ion optics was established and large mass spectrometers were designed and constructed. In 1970, we have calculated the ion trajectory in an electric and a magnetic field up to third order approximation and made a computer program TRIO $^{\text {1) }}$ using these results. This program TRIO has often been used effectively for two purpeses: the design of a new mass spectrometer $^{23}$ and the improvement of an existing mass spectrometer.

Since 1980, field desorption (FD), fast atom bombardment (FAB) and secondary ion (SI) ionization techniques have been introduced, and it becomes possible to produce molecular ions of labile compounds of several thousand daltons. The demand for new ion optics of a high performance mass spectrometer has arisen. High mass range and high transmission efficiency become important as well as the mass resolution. In order to allow high transmission keeping still high resolution, the second and third order image aberrations should be corrected by giving the special curvature at the boundary of electrode

${ }^{*}$ Institute of Physics, College of General Education. Osaka University, Toyonaka 560, Japan. 
and/or magnet or by introducing quadrupole, hexapole and/or octupole lenses. Then, the ion optical system becomes complicate and we must optimize more than ten parameters of a mass spectrometer system. It is very difficult to find the best solution by trial and error method. We have introduced one of the automatic fitting function (simplex method) and made computer program TRIO-FIT. There is already a computer code of ion optical calculation by means of the fitting function., ${ }^{3,4}$ The advantageous feature of TRIO-FIT is the capability of calculating the trajectory in an elecctric field and the flexibility of choosing any constraint equation. In the present paper, we explain the way how to use TRIO-FIT for a model mass spectrometer.

\section{Automatic Fitting Routine : Simplex Method}

Several mathematical methods have been proposed for the purpose of optimization.") Simplex code ${ }^{(5)}$ was adopted in TRIOFIT because it converges quickly froma set of rough initial values. The problem is how to define the constraint equation. In other computer codes, constraints are given in the form of a transfer matrix element. The merit of such a method is that all input data are given as "data format" and the source program need not be modified. The demerit is that we can not choose an arbitrary constraint. Then, in TRIO-FIT we adopt the method that we will write the arbitrary constraint equation explicitly in the subroutine FCN. The details will

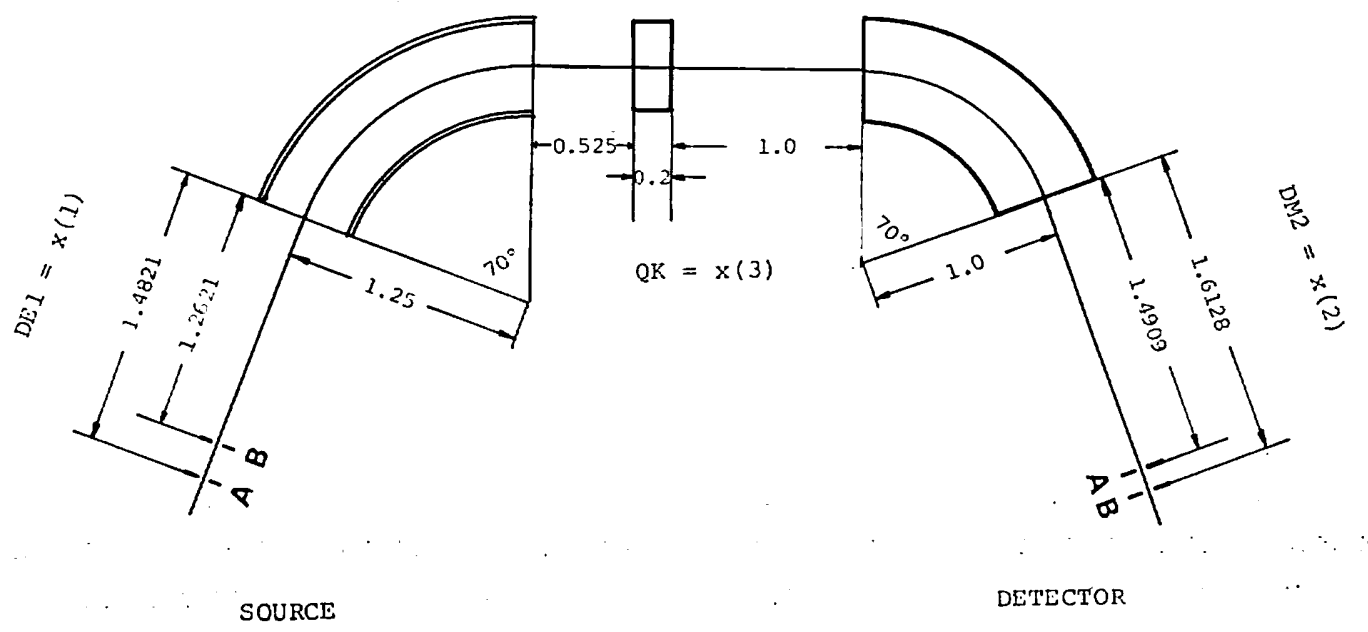

Fig. 1. The ion optical block diagram of a modified Nier-Johnson type mass spectrometer. Indication $\mathrm{A}$ means the original position of the source and detector slits (the field strength of an electric Q-lens equals to zero). Indication B means the calculated position of source and detector slits (the field strength of an electric Q-lens equals to -1.67 ). 


\section{Computer Program TRIO-FIT for Third Order Calculation of Ion Trajectory with Help}

be explained in the following example.

\section{Construction of Program}

Several modifications of the original TRIO were made in TRIO-FIT. The main program was changed and three subroutines MATRIX, FCN, SIMPLX were added. We will explain this new function by using a model system. We intend to modify a Nier-Johnson-type mass spectrometer by introducing a quadrupole lens between an electrode and a magnet in order to improve ion optical character in the axial direction. The ion optical block diagram is shown in Fig. 1. Two drift length DE1, DM2 and the field strength QK of the electric quadrupole lens are chosen as the parameters which should be determined by TRIO-FIT so as to satisfy the triple focusing condition (radial angle, axial angle and energy focusing).

\subsection{Main Program}

The main program (source program) should be prepared by the user. The preparation is quite simple as shown in appendix. The way how to write the main program for a model system is explained following to the statement number.

30- 60: Labelled COMMON statements which can never be omitted.

70- 80: DIMENSION and EXTERNAL statements for subroutine SIMPLX

90-280: Arguments for the subroutine SIMPLX.

$300-340$ : Print out of ion optical informations before fitting.

350-360: Call the subroutine SIMPLX. $370-390$ : Print out of ion optical informa- tions after fitting.

\subsection{Subroutines}

3.2.1. SIMPLX (FCN, N, X, EPSF, EPSX, ICONT, NCUT, JXD, XD, JOKEN, XMAX, XMIN, IPRINT, NMOD, FMIN, KAISU, W, NW)

The meanings of arguments are as follows.

FCN : The constraint equation. (subroutine is given in Appendix I)

$\mathrm{N}$ : Number of variables.

$\mathrm{X}$ : Components of vector $\mathrm{x}$ on $\mathrm{N}$ dimensional space. Initial values are given $\left(X(J)=V_{J}, J=1,2 \cdots N\right)$ and optimized values are given as output data.

EPSF,. When the value or the change EPSX $\}^{\text {: }}$ of constraint equation becomes less than these numbers, the search stops.

ICONT : As input parameters. ICONT $=1$ requests to search till the shape of constraint to be conede type. ICONT $\neq 1$ does not request such a condition. As output parameters. ICONT $=1$ means that the search finished after convergence. ICONT $=-1$ means that it finished without convergence.

NCUT: Maximum number of calculating the values of constraint equation. The program stops when the calculating number is bigger than this number.

$\mathrm{JXD}$. The program changes the $\mathrm{J}$ com$\mathrm{XD}\}$ : ponent of $\mathrm{X}$ by $\pm[\mathrm{XD}(\mathrm{J}) * \mathrm{X}(\mathrm{J})]$. If $\mathrm{JXD}=1, \mathrm{XD}(\mathrm{J})$ can be arbitrary given. If $\mathrm{JXO} \neq 1, \mathrm{XD}(\mathrm{J})$ $=0.1$ is always used. 


\section{T. Matsuo}

JOKEN If JOKEN $\neq 0$, the variables $X M A X\}: X(J)$ is searched between maxi$\mathrm{XMIN}$ mum value $\mathrm{XMAX}(\mathrm{J})$ and minimum value XMIN(J). If JOKEN $=0, X(J)$ has no limitation.
IPRINT . IPRINT indicates the style of NMOD $\}^{\text {' }}$ intermediate output.

IPRINT $\geq 4$ No output. IPRINT $=1,2$ short informations are printed out.

Appendi: I.

$10 C$ TRIC-FIT PROGRAM

200 MAIN PROGRAM

3C COMMON /ELOCK $1 / A(2,59)$

40 COMMON/BLOCK2/B $(2,59)$

50 COMMON /BLOCK3/NGROUP, NORDER

60 COMMON /BLOCK 4/APRINT, BPRINT, TFEINT

70 DIMENSION $X(3), X D(3), X M A X(3), X M I N(3), W(50)$

EO EXTERNAL FCN

SC $\quad \mathrm{N}=3$

$100 \quad X(1)=1.0$

$110 \quad x(2)=1.0$

$120 \quad X(3)=0.0$

130 EPSF $=1.0 \mathrm{E}-8$

$140 \quad$ EPSX $=2.0 \mathrm{E}-7$

$150 \quad$ ICONT $=0$

150 NCUT $=1000$

170 JXD $=0$

180 JOKEN $=1$

$190 \quad$ XMAX $(1)=100.0$

$200 \quad$ XMAX(2) $=100.0$

$210 \quad \operatorname{XMAX}(3)=100.0$

$220 \quad \operatorname{XMIN}(1)=0.0$

$230 \quad X M I N(2)=0.0$

$240 \quad X M I N(3)=-100.0$

250 IPRINT $=2$

260 NMOD $=5$

270

280

$\mathrm{NW}=\mathrm{N}^{*} \mathrm{~N}+8+\mathrm{N}+5$

$\mathrm{KAISU}=0$

$290 \mathrm{C}$

300

310

320

330

340

350 


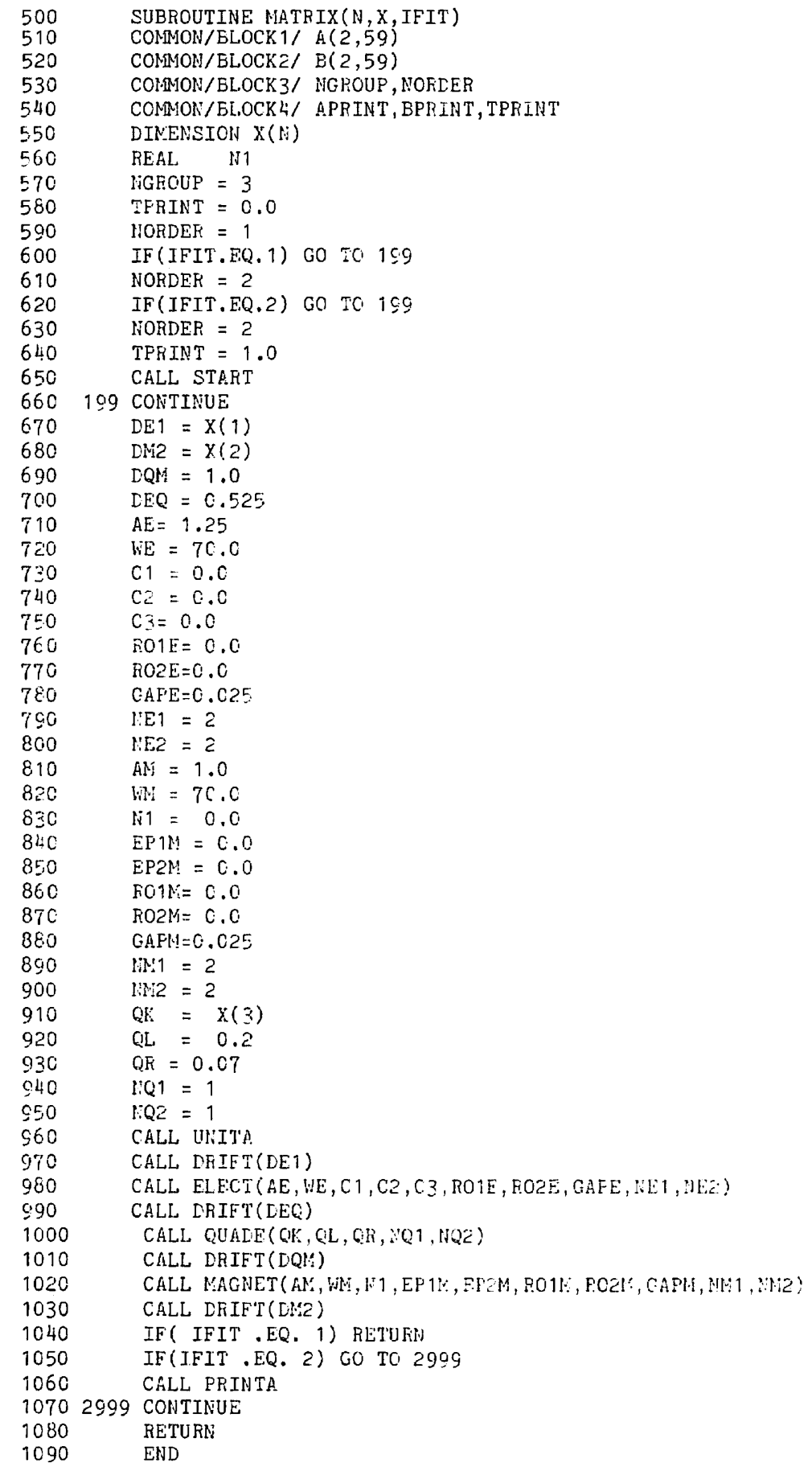


IPRINT $=1$ standard form of output. FMIN and $\mathrm{X}(\mathrm{J})$ are printed out every tenth repetition. IPRINT $=-1,-2$ Detailed information are printed out after every [NMOD] repetition.

KAISU : Number of calling the function.

FMIN : Value of optimized constraint equation.

W . working area. $N W \geq N^{2}+8 N+1$, $N W\}: W(K), K \geq N W$.

the source LIST of subroutine SIMPLX is omitted because it is too lenghty and the modification by user is unnecessary.

\subsection{2. $\mathrm{FCN}(\mathrm{N}, \mathrm{X}, \mathrm{F})$}

The meanings of arguments are as follows :

$\mathrm{F}$ : The constraint equation. $\mathrm{F}$ must be defined in this subroutine in the explicit formula like as $\mathrm{F}=\mathrm{A}(1,2) \times \mathrm{A}(1,2)+\mathrm{A}(1,4) \times$ $A(1,4)+A(1,51) \times A(1,51)$

$N$ These arguments are the same $\mathrm{X}^{\circ}$ as defined in SIMPLX.

The way how to write the subroutine FCN for a model system is explained following to the statement number.

420-450: Name of SUBROUTINE, COMMON and DIMENSION. User need not to modify these statement.

460: Calculation of transfer matrix of a system under the current variables.

470: Definition of constraint equation.

\subsubsection{MATRIX (N, X, IFIT)}

The meanings of arguments are as follows :
IFIT: This arguments defines the condition of print out format.

$=1$ NO printout of transfer matrix. IFIT must be 1 in subroutine $\mathrm{FCN}$.

$=2$ Only transfer matrix is printed out.

$=3$ All ion optical parameters and the total transfer matrix elements are printed out.

$\mathrm{N}$. These arguments are the same \}$^{*}$ : as defined in SIMPLX

The total transfer matrix of a system is calculated by this program. The arrangement of statement is almost the same as those of main program of original TRIO. The meaning of arguments of subroutines used in MATRIX are the same as original TRIO. MATRIX is usually called in FCN just before defining the constraint equation F.

The way how to write the subroutine MATRIX for a model system is explained following to the statement number.

500-550: Name of SUBROUTINE and COMMON, DIMENSION, area. User need not modify these statements.

560: REAL statement for N1.

570-660; Definition of NORDER and print out style.

670-950: Definition of element parameters of a model mass spectrometer system. It should be noted that $\mathrm{DE} 1=\mathrm{X}(1), \mathrm{DM} 2=\mathrm{X}(2)$ and $\mathrm{QK}$ $=X(3)$ are the variables which should be determined by TRIOFIT in this example.

960-1030: Arrangement of a mass spectrometer is defined and the total 


\section{Computer Program TRIO-FTI for Third Order Calculation of Ion Trajectory with Help}

transfer matrix is calculated.

1040-1090: Print out the total transfer matrix if requested.

\section{Conclusion}

We have made the computer program TRIO-FIT and explained how to use this program for a model system. The result of calculation is given in Table I and II showing that the triple focusing conditions

$$
(x \mid A)=(x \mid D)=(y \mid B)=0
$$

are satisfied. In this paper we have used
TRIO-FIT only in the first order approximation.

It was shown in other paper ${ }^{7)}$ that TRIO-FIT was very useful for the second and third order aberration correction of a high performance mass spectrometer. We are sure that TRIO-FIT is helpful for everyone who wants to design a high performance mass spectrometer. The program is written in FORTRAN IV and is available in the form of a magnetic tape.

Table I. OUTPUT of TRIO-FIT of a model mass spectrometer before fitting. Initial values of drift lengths (DE1, DM2) and $Q$-lens strength $(\mathrm{QK})$ are 1.0,1.0 and 0.0, respectively

BEFORE FIT SIMPLEX

ION TRAJECTORY CALCULATION BY "I F I $O$

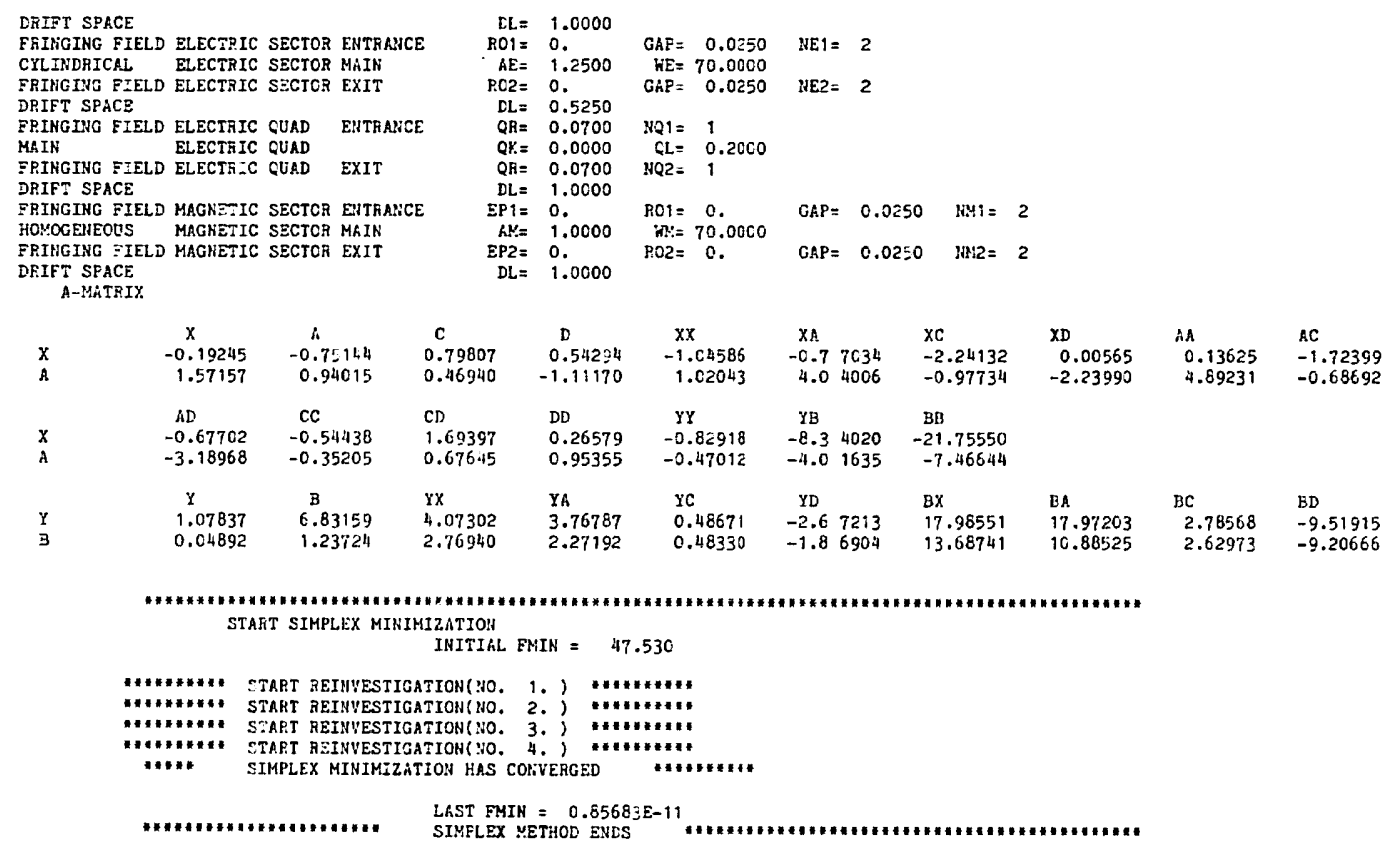




\section{T. Matsuo}

Table II. OUTPUT of TRIO-FIT of a model mass spectrometer after fitting AFTER FIT SIMPLEX

ION TRAJECTORY CALCULATION BY "T $\mathrm{P}$ I $O$ "

SECOND ORDER

MASS AHD ENERGY DISPERSIOH:

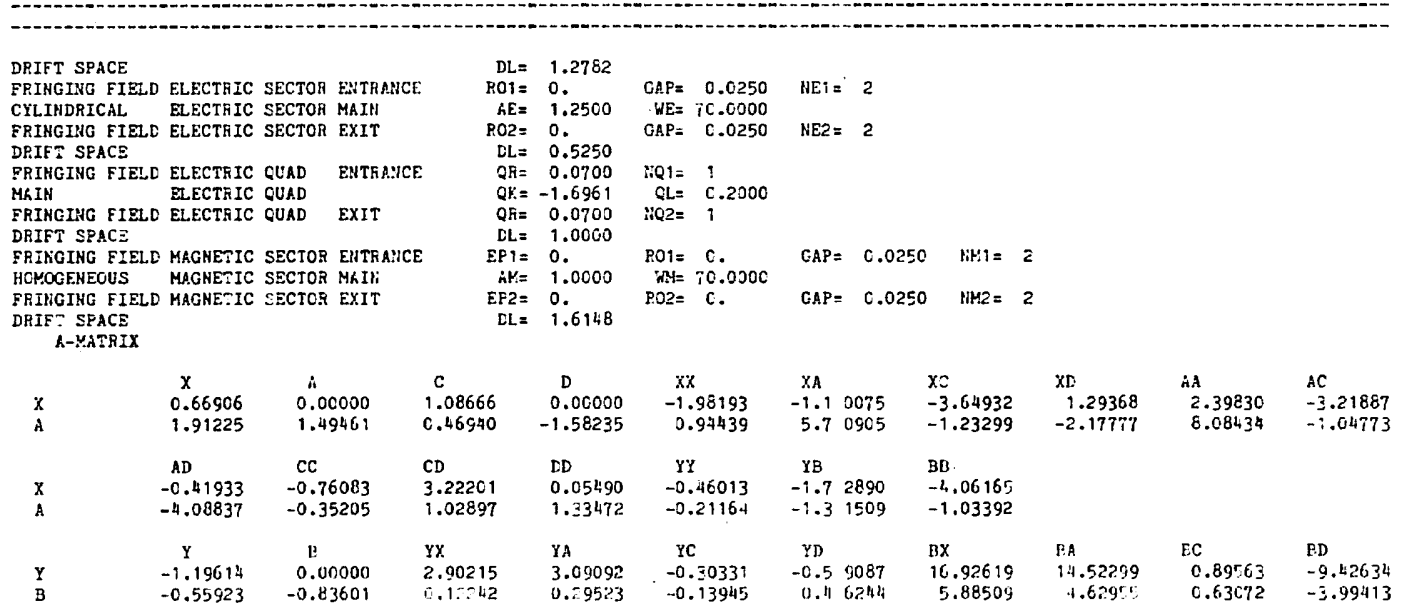

\section{Acknowledgement}

The author is grateful to Prof. $\mathrm{H}$. Matsuda for his valuable discussions and comments. He is also grateful to Prof. T. Kotani for offering the Simplex code.

\section{References}

1) T. Matsuo, H. Matsuda, Y. Fujita, and H. Wollnik, Mass Spectroscopy (Japan), 24, 19 (1975).

2) H. Matsuda, Mass Spectro. Rev., 2, 299(1983)-

3) K. L. Brown, F. Rothacker, D. C. Carey, and Ch. Iselin, SLAC Report No. 91 (1977).
4.) H. Wollnik, J. Brezina, M. Berz, and W. Wendel, At. Mass Fundam. Constants. (1984) in press.

5) J. Kowalik and M. R. Osborne, Methods for Unconstrainted Optimization Problems(American Elsevier Publishing Co., New York 1968).

6) T. Kotani, Bull. of Computation Center Osaka Univ. 33, 62 (1979).

7) T. Matsuo, Jpn. J. Appl. Phys., 23, 854 (1984).

\section{Keywords}

Ion optics

Computer program

Automatic fitting 